\title{
Effect of Dietary Nitrate Supplementation on Sleep in Chronic Obstructive Pulmonary Disease Patients
}

\author{
Jonathan P Wisor (D) \\ Nils Henrik Holmedahl (iD ${ }^{2}$ \\ Ingvild West Saxvig ${ }^{3}$ \\ Odd-Magne Fjeldstad ${ }^{2}$ \\ Eddie Weitzberg ${ }^{4}$ \\ Janne Grønli ${ }^{5}$ \\ Harald Kåre Engan ${ }^{6}$ \\ 'Sleep and Performance Research Center \\ and Department of Biomedical Sciences, \\ Elson S. Floyd College of Medicine, \\ Washington State University, Spokane, \\ WA, USA; ${ }^{2}$ Section of Lung \\ Rehabilitation, Department of \\ Rehabilitation and Lifestyle Medicine, \\ LHL-Hospital Gardermoen, Jessheim, \\ Norway; ${ }^{3}$ Norwegian Competence \\ Center for Sleep Disorders, Haukeland \\ University Hospital, Bergen, Norway; \\ ${ }^{4}$ Department of Physiology and \\ Pharmacology, Karolinska Institutet and \\ Department of Perioperative Medicine \\ and Intensive Care, Karolinska Hospital, \\ Stockholm, Sweden; ${ }^{5}$ Department of \\ Biological and Medical Psychology, Faculty \\ of Psychology, University of Bergen, \\ Bergen, Norway; ${ }^{6}$ Unicare Norway, Oslo, \\ Norway
}

This article was published in the following Dove Press journal: Nature and Science of Sleep

Purpose: Poor sleep quality in chronic obstructive pulmonary disease (COPD) is a result of oxygen desaturation secondary to compromised lung function. Nitrate supplementation with dietary beetroot juice is known to elevate plasma nitrate and to increase the efficiency of oxygen utilization in non-COPD individuals; whether it is of therapeutic benefit for sleep quality in COPD has not been reported.

Patients and Methods: In a counterbalanced within-subjects design involving 15 COPD patients as subjects, the subjects consumed either beetroot juice containing nitrate (BJ; $~ 6.2$ mmol $\left.\mathrm{NO}_{3}{ }^{-}\right)$or placebo $\left(\mathrm{NO}_{3}{ }^{-}\right.$-depleted juice) immediately before a night of polysomnographic monitoring. Nitrate was measured in plasma collected immediately after waking.

Results: While BJ consumption had no effect on the amount of time spent in any sleep stages, wake-to-N2 transitions and direct wake-to-rapid eye movement sleep (REMS) transitions, hallmarks of disordered sleep, were less frequent on the BJ night than on the placebo night. In the last two hours of the BJ night, percent time in REMS increased and delta power during deep (N3) non-REMS decreased, relative to the placebo night. Collectively, the reduced frequency of atypical transitions and the normalization of non-REMS/REMS dynamics after BJ are indicative of an improvement of sleep quality. BJ also resulted in sustained elevation of peripheral oxygen saturation $\left(\mathrm{SpO}_{2}\right)$, during episodes of wake after sleep onset. Plasma nitrate was elevated nearly tenfold on the morning after BJ relative to placebo.

Conclusion: BJ has a normalizing effect on disordered sleep in COPD, which may be related to improved oxygen delivery.

Clinical Trial Registration: The activities of the Regional Committees for Medical and Health Research Ethics (REC) are founded on the Norwegian law on research ethics and medical research. This study was approved by NTNU/REK midt, Det medisinske fakultet, Postboks 8905, 7491 Trondheim (REK midt 2016/1360).

Keywords: polysomnography, oxygen saturation, beetroot juice, hypoxia

\section{Plain Language Summary}

Chronic obstructive pulmonary disease is characterized by disrupted sleep due to low blood oxygen levels during sleep. Dr. Wisor and colleagues sought to improve sleep in COPD patients by giving them a dietary supplement, beetroot juice. Beetroot juice contains nitrate, a substance that is known to elevate blood oxygen levels and improve oxygen delivery to tissues. When COPD patients drank beetroot juice at bedtime, their overnight sleep quality improved. The duration of deep sleep episodes was increased on the night when patients drank beetroot juice at bedtime. The frequency of events indicative of healthy sleep,
Correspondence: Jonathan P Wisor

Washington State University, 4 I 2 E.

Spokane Falls Blvd, Spokane, WA, 99202,

USA

Tel + I 509358-7577

Email Jonathan.Wisor@wsu.edu 
transitions from sleep of immediate depth to deeper sleep, increased on the night when patients drank beetroot juice at bedtime. The electroencephalographic brain waves measured as part of the study showed an increase in slow oscillatory activity on the night when patients drank beetroot juice at bedtime, a sign of increased sleep depth. Additionally, the blood oxygenation level increased on the night when patients drank beetroot juice at bedtime, albeit only during periodic intervals of awakening from sleep.

\section{Introduction}

COPD often requires the use of accessory muscles to overcome inadequate ventilation. ${ }^{1,2}$ The activity of these voluntary muscles is suppressed during sleep, resulting in insufficient ventilation, increased arterial carbon dioxide, oxygen $\left(\mathrm{O}_{2}\right)$ desaturation and disruption of sleep ${ }^{3-5}$ Sleep efficiency (SE, percentage of time spent asleep between lights-off and lightson), total sleep time (TST) and rapid eye movement sleep (REMS) as percent of time spent asleep are all reduced in COPD patients relative to age-matched controls. ${ }^{3,4}$

Nitric oxide (NO) is a pluripotent signaling radical, involved in many diverse physiological processes including vasoregulation, nerve transmission and host defense. ${ }^{6}$ Nitrate $\left(\mathrm{NO}_{3}{ }^{-}\right)$administered via oral supplementation serves as a substrate for the systemic generation of NO, which prophylactically supports physiological adaptations to hypoxia, including vasodilation and changes in aerobic respiration rate. $^{6}$ Dietary supplementation with beetroot juice $(\mathrm{BJ}$; a natural source of inorganic nitrate) reduces $\mathrm{O}_{2}$ consumption during exercise in healthy subjects. ${ }^{7}$ Its effects on the performance of chronic obstructive pulmonary disease patients in exercise are equivocal. ${ }^{8-11}$ However, reductions in resting blood pressure after BJ consumption by COPD patients ${ }^{9,11}$ indicate potential benefits in the resting state.

The role of NO in cerebral function is complex and has been described as a double-edged sword since it is involved in normal physiology as well as in pathophysiological processes. All three known NO synthase isoforms can be expressed in the brain. Among many functions NO is involved in learning and memory, ${ }^{12}$ cerebral autoregulation ${ }^{13}$ and neurovascular coupling $^{14}$ via modulation of nerve transmission both at the post- and presynaptic levels. Moreover, from studies using NO synthase inhibitors or NO donors it has become evident that NO is implicated in modulation of sleep-wake states. ${ }^{15}$ Contrary, NO generation is highly stimulated during excitatory signaling via the glutamate-NMDA pathway, but the roles of NO in conditions such as brain ischemia and seizures are still not clear ${ }^{16}$ Only a few studies have investigated the effect of nitrate supplementation on cerebral physiology. It has been shown that dietary nitrate may increase cerebral blood flow ${ }^{17,18}$ and some studies show improved cognitive function ${ }^{17,19}$ after supplementation with BJ. In a repeated measured experiment involving 15 COPD patients, we assessed the effect of BJ containing nitrate on nighttime sleep quality and efficiency.

\section{Materials and Methods Subjects}

From September 2016 to June 201715 subjects were recruited while attending a four-week, in-patient pulmonary rehabilitation program at the LHL-hospital, Glittre, Norway. Prior to inclusion, a diagnosis of COPD in a stable state was verified by pulmonary function tests, see Table 1 . Most had moderate or severe COPD - Global Initiative for Chronic Obstructive Lung Disease (GOLD) severity stage II and III-one had mild COPD (GOLD stage I) and three patients had very severe COPD (GOLD stage IV). All used inhaler medication in accordance with the GOLD recommendations. None of the patients used supplementary oxygen, all were former smokers except one who was currently smoking with a carboxyhemoglobin level of $4.3 \%$. All had at least one comorbidity, of which the most common were arthrosis or rheumatic disease (nine patients) and hypertension (five patients), none had confirmed asthma overlap with COPD. Demographic details of the study population are published elsewhere ${ }^{20}$ and are replicated here in Table 1. Exclusion criteria were COPD exacerbation within the last three weeks, chronic respiratory failure, other diseases significantly affecting upper and lower airway function, a prior diagnosis of OSA, non-sinus cardiac arrhythmias, implanted pacemaker, coronary arterial disease with unstable angina pectoris or myocardial infarction within the last three months, uncontrolled hypertension, history of cerebral infarction, use of nitrate medication. All subjects used prescribed medication, but no respiratory depressant drugs were taken from $48 \mathrm{~h}$ prior to first PSG-recording until end of study. Informed consent was obtained from all individual subjects included. All procedures performed were in accordance with the 1964 Declaration of Helsinki and its later amendments. The study was approved by the regional ethics committee (2016/ 1360/REK Midt, NTNU/REK midt, Det medisinske fakultet, Postboks 8905, 7491 Trondheim).

\section{Dietary Beetroot Juice Administration}

BJ containing nitrate $\left(70 \mathrm{~mL}, \sim 6.2 \mathrm{mmol} \mathrm{NO}_{3}{ }^{-}\right.$; James White Drinks, Ltd) or placebo (70 $\mathrm{mL}, \mathrm{NO}_{3}{ }^{-}$depleted juice) was administered orally immediately before bedtime on nights two 
Table I Study Population

\begin{tabular}{|c|c|}
\hline & $\mathrm{N}=15$ Mean $(95 \% \mathrm{Cl})$ \\
\hline \multicolumn{2}{|l|}{ Demographic data } \\
\hline Gender female & $8(53)^{\mathrm{a}}$ \\
\hline Age, years & $60.7(55.8-65.7)$ \\
\hline BMI, $\mathrm{kg} / \mathrm{m}^{2}$ & $26.1(23.2-29.1)$ \\
\hline \multicolumn{2}{|l|}{ Smoking habit } \\
\hline Former smoker & $15(100)$ \\
\hline Current smoker & I $(7)^{a}$ \\
\hline \multicolumn{2}{|l|}{ Spirometry } \\
\hline FVC, L & $3.3(2.8-3.7)$ \\
\hline FEVI, L & $1.4(1.1-1.7)$ \\
\hline FEVI/FVC, ratio & $0.42(0.35-0.49)$ \\
\hline DLCO, \% pred & $54.1(43.2-64.9)$ \\
\hline $\mathrm{RV} / \mathrm{TLC}$, ratio & $0.54(0.48-0.60)$ \\
\hline \multicolumn{2}{|l|}{ Clinical data } \\
\hline GOLD stage & $2.7(2.2-3.2)$ \\
\hline CAT, score & $16.5(12.4-20.7)$ \\
\hline MMRC, score & I.8 (I.2-2.4) \\
\hline 6-MWD, meter & $426(364-488)$ \\
\hline ESS score & $5.0(3.7-6.3)$ \\
\hline \multicolumn{2}{|l|}{ Laboratory data } \\
\hline $\mathrm{P}_{\mathrm{a}} \mathrm{O}_{2}, \mathrm{kPa}$ & $9.13(8.56-9.7 I)$ \\
\hline $\mathrm{P}_{\mathrm{a}} \mathrm{CO}_{2}, \mathrm{kPa}$ & $4.98(4.64-5.32)$ \\
\hline $\mathrm{COHb}, \mathrm{b} \%$ & $1.4(1.3-1.6)$ \\
\hline $\mathrm{S}_{\mathrm{a}} \mathrm{O}_{2},{ }^{\mathrm{c}} \%$ & 96.1 (95.1-97.2) \\
\hline
\end{tabular}

Notes: ${ }^{\mathrm{a}} \mathrm{N}$ (\% of study subjects), instead of mean $(95 \% \mathrm{Cl}) .{ }^{\mathrm{b}} \mathrm{N}=12$, two missing and one current smoker excluded from $\mathrm{COHb}$ analysis. ${ }^{\mathrm{C}} \mathrm{N}=14$, one missing from $\mathrm{S}_{\mathrm{a}} \mathrm{O}_{2}$. Abbreviations: BMI, body mass index; FVC, forced vital capacity as liters; FEVI, forced expiratory volume first second as liters; DLCO, diffusing capacity of the lung for carbon monoxide as percent of predicted value; RV, residual volume of the lungs; TLC, total lung capacity; GOLD stage, Global Initiative for Chronic Obstructive Lung Disease severity stage; CAT, COPD assessment test; MMRC, Modified Medical Research Council questionnaire; 6MWD, six minutes walking distance; $\mathrm{P}_{\mathrm{a}} \mathrm{O}_{2}$, arterial pressure of oxygen; $\mathrm{P}_{\mathrm{a}} \mathrm{CO}_{2}$, arterial pressure of carbon dioxide; $\mathrm{S}_{\mathrm{a}} \mathrm{O}_{2}$, arterial oxygen saturation; $\mathrm{COHb}$, carboxylated hemoglobin.

and three of the study in a double-blind, counterbalanced design. This dose has been used in several studies to achieve effects on blood pressure and oxygen cost during exercise. ${ }^{7,21}$ The placebo was prepared from the BJ in a previously described nitrate-depletion protocol that leaves the liquid identical in taste to the original $\mathrm{BJ} .{ }^{19,21}$

\section{Polysomnography}

Subjects slept three nights semi-unattended in their hospital room, with PSG recorded online via a bedside computer. The first night served as an adaptation night, for acquaintance with the equipment (no recording was done). The second and third nights were block randomized to nitrate intervention or control (placebo). Patients were block randomized according to sex and age. Randomization was performed using a web-based randomization system developed and administered by the Unit of Applied Clinical Research at the Norwegian University of Science and Technology-NTNU in Trondheim, Norway. Randomization was undertaken in a 1:1 ratio. The nurse reported time of lights off. PSG was recorded by Embla A10 (Medcare Flaga, Reykjavik, Iceland) according to the 2007 recommendations from the American Academy of Sleep Medicine (AASM), ${ }^{22}$ including electroencephalogram (F4$\mathrm{M} 1, \mathrm{C} 4-\mathrm{M} 1, \mathrm{O} 2-\mathrm{M} 1$ ), left and right electrooculogram, and electromyogram submentalis monitoring. In addition, an electromyography of anterior tibialis, thoracic and abdominal breathing movements/respiratory effort, nasal airflow pressure, $\mathrm{SpO}_{2}$, and body position were measured. All signals were sampled using Somnologica Studio Version 3.3 software (Medcare Flaga).

\section{Polysomnographic Data Analysis}

All subjects spent at least $6.2 \mathrm{~h}$ in bed in both treatment conditions; the data analysis was therefore restricted to the first six hours in bed. Sleep was classified by trained experts in 30-second epochs as wake, non-REMS stages 1-3 (N1, N2, $\mathrm{N} 3$ ) or REMS according to the rules set by the AASM. ${ }^{23}$ Wake was further sub-classified as quiet wake or active wake algorithmically: wake epochs in which the root mean square value of submental muscle tone was less than the thirty-third percentile for all wake epochs was classified as quiet wake (QW); wake epochs in which the root mean square value of submental muscle tone was more than the sixty-sixth percentile for all wake epochs was classified as active wake (AW); epochs falling between the thirty-third and sixty-sixth percentiles were referred to as intermediate wake (IW). Fast Fourier transformation was used to calculate electroencephalographic power for each stage in $1 \mathrm{~Hz}$ bands from 1 to $20 \mathrm{~Hz}$. In addition, power in the delta $(1-4 \mathrm{~Hz})$, theta $(5-8 \mathrm{~Hz})$, alpha (9-12 Hz), and beta (15-35 Hz) ranges was calculated for each stage from each of two EEG derivations (F4:M1, C4:M1).

\section{Plasma Nitrate and Nitrite Measurement}

Plasma nitrate and nitrite measurements were performed on samples from 14 of the subjects, using methods described previously. ${ }^{21}$ Venous blood samples were collected in the morning immediately after termination of the PSG protocol. Plasma was isolated by centrifugation and stored at $-80^{\circ} \mathrm{C}$. Plasma content of nitrate and nitrite were measured using a HPLC system. ${ }^{24}$ 


\section{Statistical Analyses}

Statistics were performed with Statistica version 13.3. All variables were assessed for normality using the Kolmogorov-Smirnov D statistic. In the cases where $P<0.05$ for the D statistic, the nonparametric Wilcoxon's matched pairs test was applied (eg frequencies of sleep stage transitions, as noted in the results section). In the cases where $P>0.05$ for the $\mathrm{D}$ statistic, normality was assumed and parametric statistics were applied.

Although time $\dot{x}$ treatment interaction is hypothesized in the analysis of EEG spectral variables, the statistical power of time $\dot{x}$ treatment interaction is limited for EEG analysis because of missing values. For instance, only six of 15 subjects entered REMS in the first two hours of both the placebo night and the nitrate night; repeated measures ANOVA across entire night on EEG parameters would be restricted to only these six subjects despite the fact that all 15 exhibited REMS later. Therefore, we performed separate comparisons of EEG spectral variables independently within each two-hour interval, and did not incorporate time of night as a repeating variable, to maximize sample size and statistical power. Time of night effects were thus inferred, though not statistically quantified per se, when one two-hour interval but not another exhibited a treatment effect.

Polysomnographic sleep timing variables known to be modulated homeostatically as a function of sleep need/time spent asleep were segmented into three twohour intervals. These variables were subjected to nested repeated measures ANOVA with interval (two-hour interval) nested within night (BJ vs placebo).

When ANOVA yielded significant effect of night only, data from the entire six-hour recording interval were subjected to post hoc Student's $t$-test for paired samples. When ANOVA yielded significant night $\dot{x}$ interval interaction, data from each two-hour interval were subjected to post hoc Fisher's least significant difference test of $\mathrm{BJ}$ vs placebo night data. Other polysomnographic variables (those that were not subjected to segmentation during the night) and nitrate/nitrite values were subjected to Student's $t$-test for paired samples.

Alpha values for the threshold of statistical significance were set at 0.05 . Measures of variability reported are standard error of the mean.

\section{Results}

\section{Plasma Nitrate and Nitrite}

Morning plasma nitrate (Figure 1A) was elevated nearly tenfold by BJ $(269 \pm 17 \mu \mathrm{M})$ relative to placebo $(27 \pm 3 \mu \mathrm{M}$;
$P<0.001$, Student's $t$-test). Morning plasma nitrite (Figure 1B) was unaffected by BJ relative to placebo.

\section{Oxygen Saturation $\left(\mathrm{SpO}_{2}\right)$}

$\mathrm{SpO}_{2}$ was highly dependent on sleep state $\left(\mathrm{F}_{2,20}=29.6\right.$, $P<0.001) . \mathrm{SpO}_{2}$ was highest in wake, intermediate in NREMS sleep stages and lowest in REMS (Figure 2),

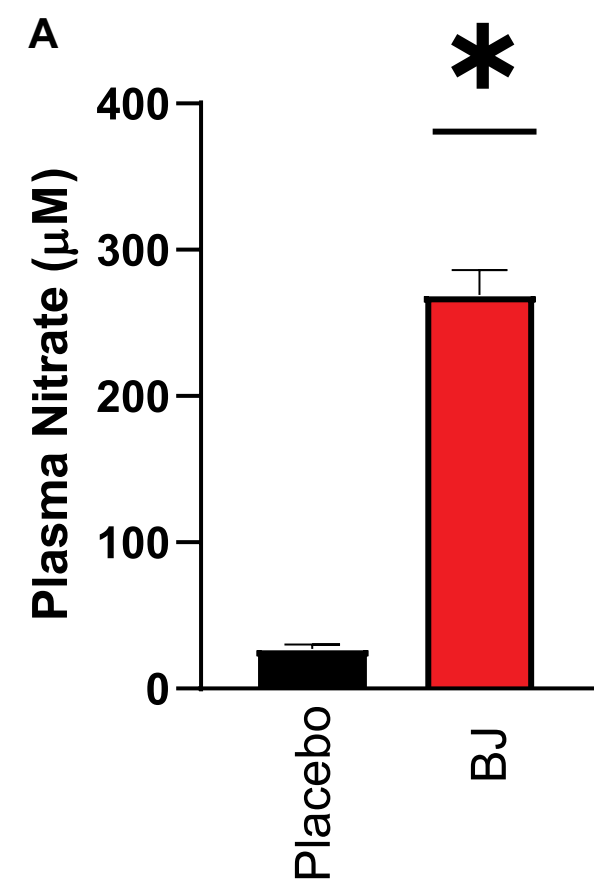

B

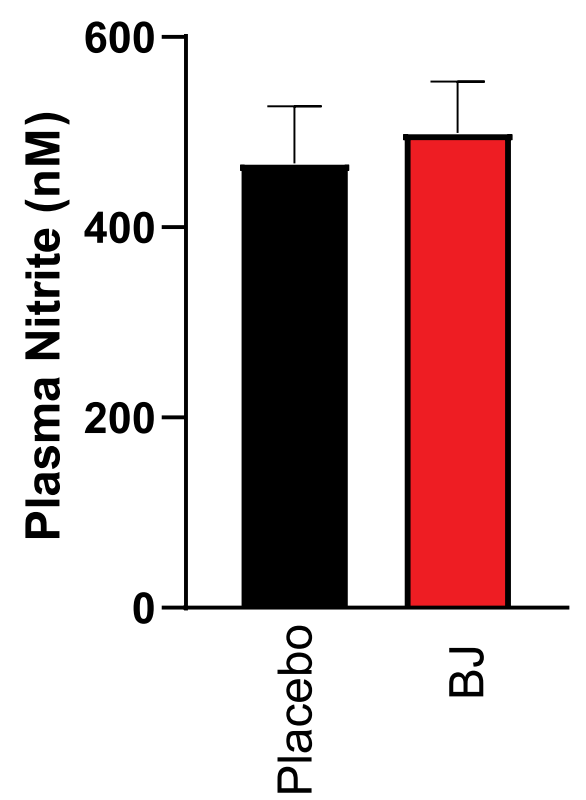

Figure I Morning plasma nitrate (A) and nitrite (B). ${ }^{*} P<0.05$ beetroot juice (BJ) vs placebo, Student's t-test. 


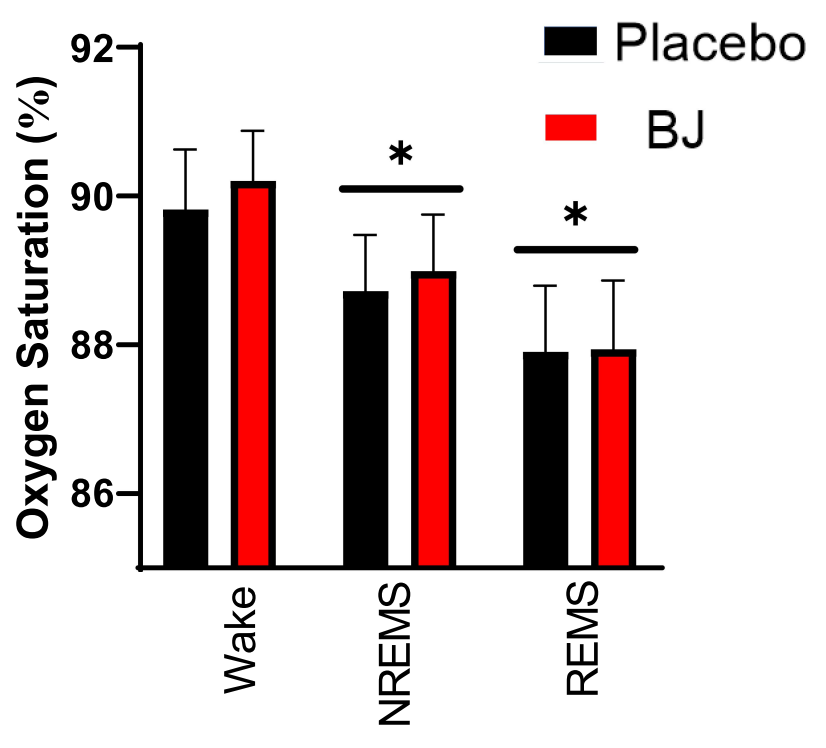

Figure 2 Sleep state dependence of oxygen saturation. $* P<0.01$ vs wake in the same treatment for both beetroot juice $(B J)$ and placebo, Fisher's least significant difference.

irrespective of treatment. The intra-episode dynamics of $\mathrm{SpO}_{2}$ (Figure 3) demonstrated state-specific temporal profiles. There was a significant effects of time on $\mathrm{SpO}_{2}$ in wake $\left(\mathrm{F}_{9,99}=2.2, P=0.025\right)$, NREMS $\left(\mathrm{F}_{9126}=3.7, P<0.001\right)$ and REMS $\left(\mathrm{F}_{9117}=3.4, P<0.001\right)$. In both NREMS (Figure 3B) and REMS (Figure 3C), $\mathrm{SpO}_{2}$ declined from the onset of the episode and remained significantly below the value at episode onset for at least five minutes. By contrast, wake onset was characterized by an immediate rise in $\mathrm{SpO}_{2}$ that reversed over time (Figure 3A). The interval over which $\mathrm{SpO}_{2}$ remained elevated above WASO values was extended $43 \%$ by BJ treatment relative to placebo $(P=0.028$; Figure 3D).

\section{Waking EEG}

The waking EEG was significantly modified in a frequency-specific manner by $\mathrm{BJ}$ (treatment $\mathrm{X}$ frequency interaction; Figure 4) in the F4:M1 derivation during the first two hours of recording $\left(\mathrm{F}_{19,266}=1.7, P=0.037\right)$. BJ also modified the waking EEG in the C4:M1 derivation during both hours $1-2\left(\mathrm{~F}_{19,266}=3.6, P<0.001\right)$ and hours three to four $\left(\mathrm{F}_{19,171}=5.3, P<0.001\right)$. These effects of $\mathrm{BJ}$ were predominantly in the slow wave range: post hoc comparisons at $1 \mathrm{~Hz}$ intervals indicated significant reductions in waking EEG power on the $\mathrm{BJ}$ night relative to the placebo night only at frequencies less than 5 $\mathrm{Hz}$ (Figure 4A, B and D).

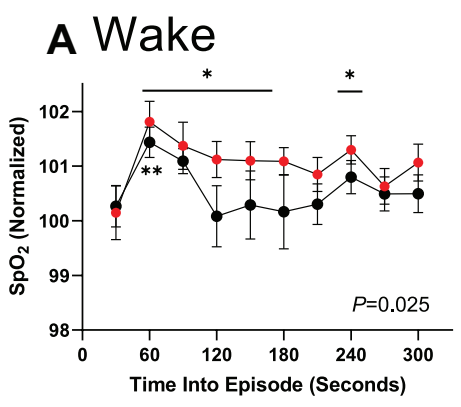

\section{B NREMS}

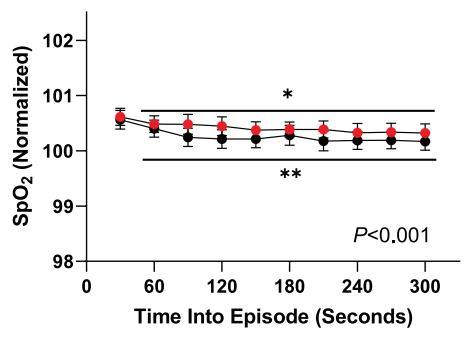

\section{REMS}
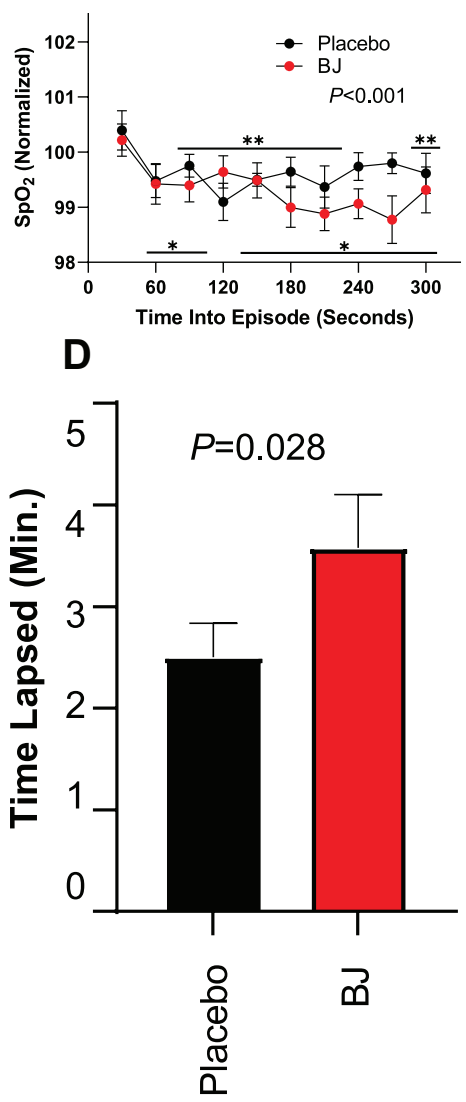

Figure 3 Changes in oxygen saturation across episodes of wake, NREMS and REMS. (A-C) Data are the average value from each 30-second interval of all episodes of wake (A), NREMS (B) or REMS (C) of at least five-minute duration, and are normalized as a percentage of the average $\mathrm{SpO}_{2}$ value across the entire night. $P$-values refer to main effect of time in repeated measured ANOVA. $* P<0.05$ $B J$ vs $B J$ value at episode onset, Fisher's LSD. $* * P<0.05$ placebo vs placebo value at episode onset, Fisher's LSD. (D) Time lapsed until $\mathrm{SpO}_{2}$ values dropped below value at wake onset. $P$-value in (D) refers to Wilcoxon's matched pairs test, beetroot juice (BJ) vs placebo. 

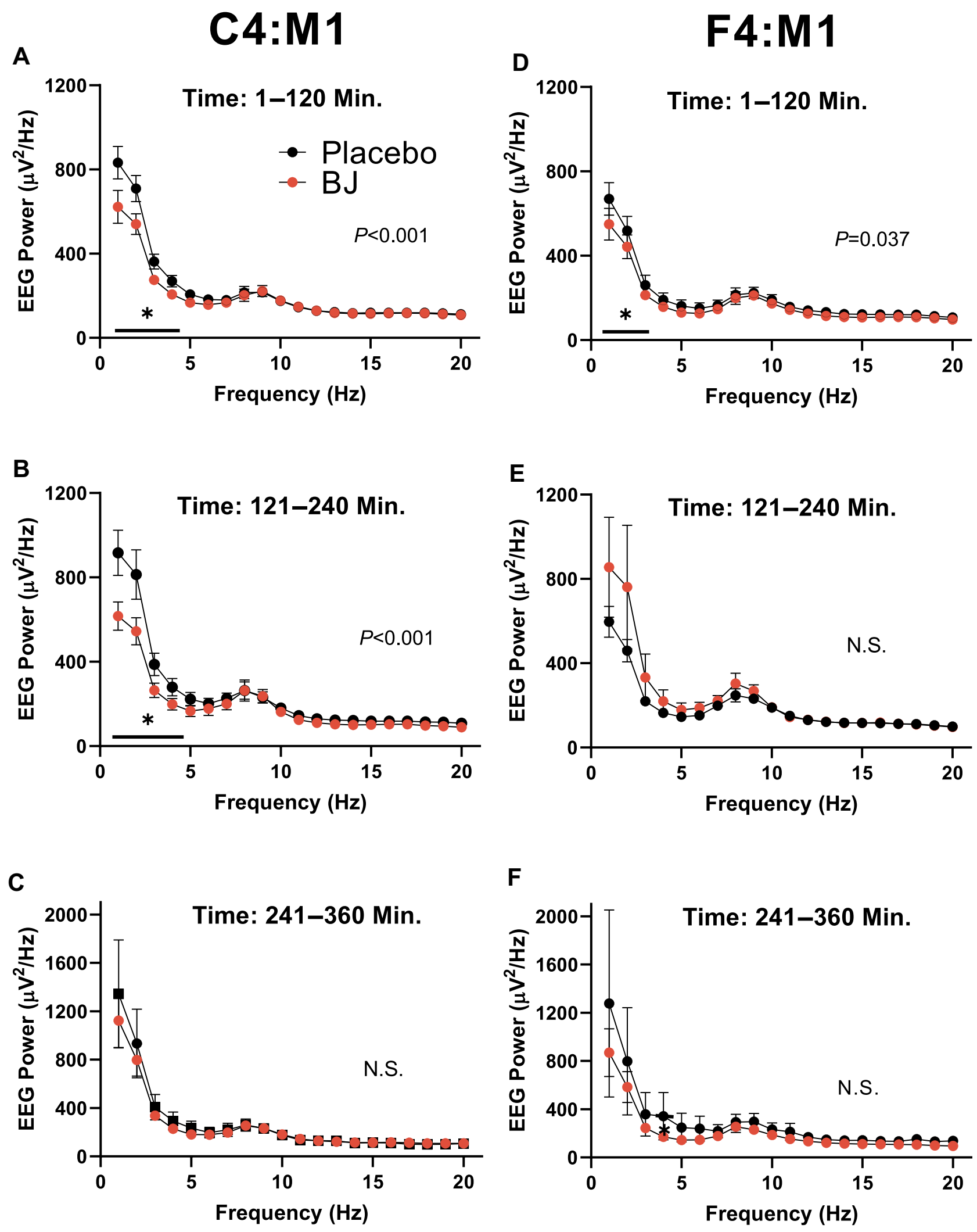

Figure 4 EEG spectral power during wake. (A-C) Data from the C4:MI derivation in the first, second, and third two-hour intervals (D-F) Data from the F4:MI derivation in the first, second, and third two-hour intervals. $P$-values refer to treatment $\dot{x}$ frequency interaction in repeated measured $A N O V A$. $* P<0.05$ beetroot juice (BJ) vs placebo value at same frequency, Fisher's LSD. Abbreviation: N.S., not significant. 
Table 2 Time Spent in Wake and Sleep States Across the Entire Six-hour Recording Session. There Were No Significant Effects of Treatment on Time Spent in Any State

\begin{tabular}{|l|l|l|}
\hline State & Placebo (Min./Six Hours) & BJ (Min./Six Hours) \\
\hline Active wake & $27 \pm 6$ & $27 \pm 6$ \\
Intermediate wake & $30 \pm 6$ & $26 \pm 6$ \\
Quiet wake & $29 \pm 6$ & $27 \pm 7$ \\
NI & $49 \pm 6$ & $40 \pm 5$ \\
N2 & $144 \pm 10$ & $143 \pm 11$ \\
N3 & $37 \pm 8$ & $46 \pm 11$ \\
REM & $44 \pm 5$ & $51 \pm 5$ \\
\hline
\end{tabular}

\section{Sleep EEG}

Polysomnography indicated no effect of BJ on the total amount of time spent in any sleep stages. For all states other than REMS, this was true effect whether the data were considered across the entire night as a main effect of treatment (Table 2), or segmented into two-hour intervals to observe time-in-bed dependent changes in sleep (Figure 5). Time spent in REMS exhibited a time $\dot{x}$ treatment interaction $\left(\mathrm{F}_{2,28}=7.7, \quad P=0.002\right)$. REMS increased across two-hour intervals in the BJ condition but was reduced in the third two-hour interval relative to the second in the placebo condition (Figure 5E).

Effects of BJ on sleep architecture were addressed by measuring the frequencies of sleep stage transitions (Table 3 ) and the durations of episodes. Wake-to-N2 transitions were greater than twofold more frequent after placebo (a total of 21 observed) than BJ (nine observed), resulting in a significant effect of treatment ( $P=0.034$, Wilcoxon's matched pairs test). Direct wake-to-REMS transitions were observed after placebo, but did not occur at all on nights when BJ was administered ( $P=0.021$, Wilcoxon's matched pairs test; Table 3). Conversely, transitions from NREMS to REMS occurred at increased frequency after BJ relative to placebo ( $P=0.033$, Wilcoxon's matched pairs test). NREMS bout duration was increased by approximately $15 \%$ on the night of BJ administration (20.9.8 $\pm 2.0 \mathrm{~min}$ ) relative to the placebo $\left(18.3 \pm 1.6 \mathrm{~min}\right.$; treatment effect $\left.\mathrm{F}_{1,11}=5.2, \quad P=0.044\right)$. Durations of wake and REMS bouts were not significantly affected by treatment.

EEG spectral power (1-20 Hz range) was affected by BJ only during deep slow wave (N3) sleep (main effect of BJ vs placebo treatment $\left(\mathrm{F}_{1,8}=6.2, P=0.037\right)$ and in no other sleep state. This effect of BJ on EEG power during N3 was frequency-specific and emerged over time in bed (Figure 6). Delta $(<5 \mathrm{~Hz})$ activity was reduced in the C4:M1 derivation

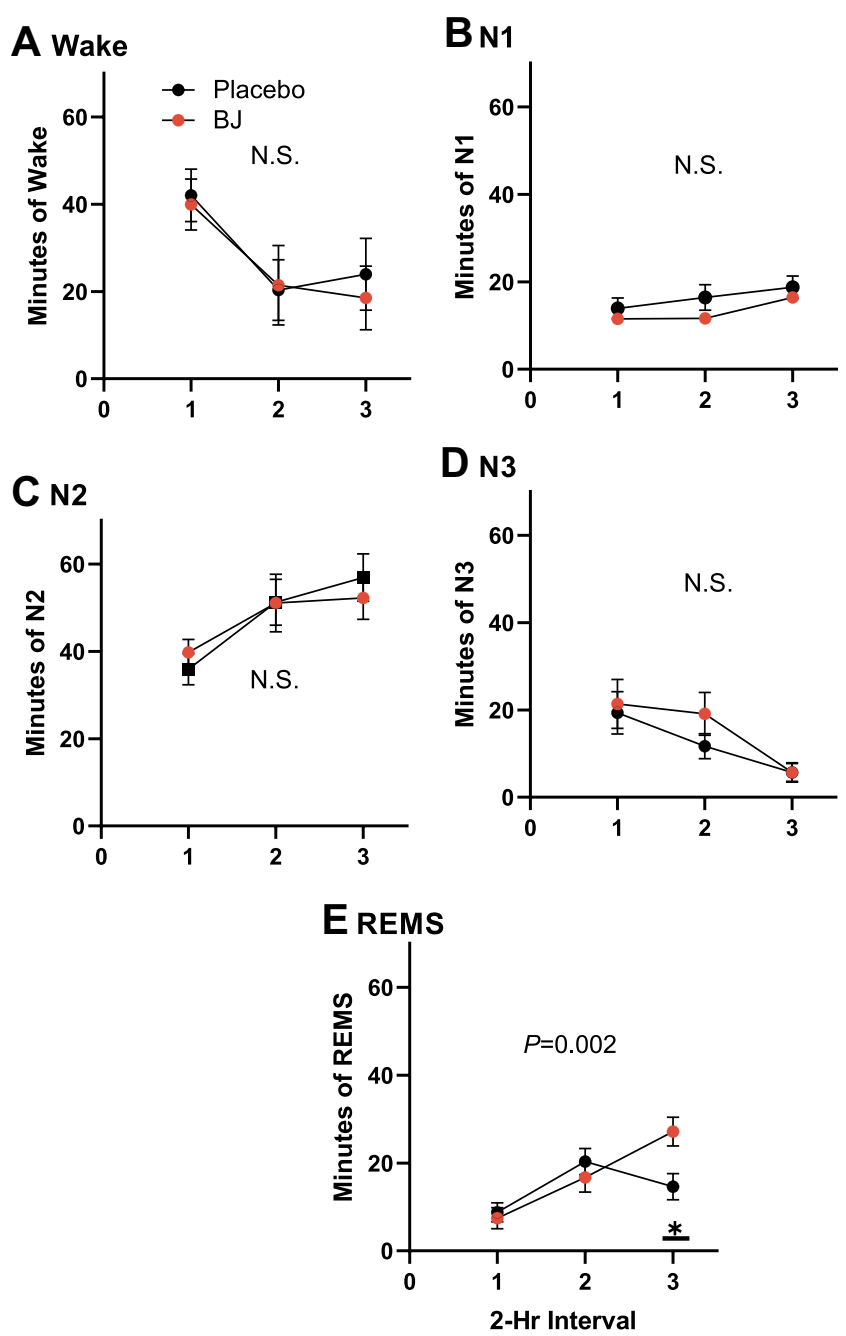

Figure 5 Minutes spent in each state across two hour intervals: (A) wake; (B-D) NI, N2, N3 non-REMS; (E) REMS. $P$-values refer to treatment $\dot{x}$ time interaction in repeated measured ANOVA. ${ }^{*} P=0.003$, beetroot juice $(B J)$ vs placebo value at same time, Fisher's LSD.

Abbreviation: N.S., not significant.

during the third two-hour interval (treatment $\dot{\mathrm{x}}$ frequency interaction $\mathrm{F}_{19,38}=2.4, P=0.012$; Figure $6 \mathrm{C}$ ). Delta activity was similarly reduced in the F4:M1 derivation during the third two-hour interval $\left(\mathrm{F}_{19,38}=2.7, P=0.004\right.$; Figure $\left.6 \mathrm{~F}\right)$. While N3 EEG spectra were equal in the first four hours of time in bed across nights, the average EEG power during N3 epochs recorded in the third two-hour interval was lower on the $\mathrm{BJ}$ night than the placebo night (Figure 6C and F). Lower delta power at the end of the BJ sleep session specifically may be a result of increased rate of slow wave sleep need discharge in the BJ condition. To address this possibility, we measured the EEG energy in the delta (1-4 Hz) range: EEG power in N3 (as reported in Figure 6) times number of minutes spent in N3 (as reported in Figure 5D). The cumulative delta energy discharged across the entire six-hour recording was significantly 
Table 3 Wake and Sleep State Transition Counts. The Values in Last Column are P-values from the Wilcoxon's Matched Pairs Test of Beetroot Juice (BJ) vs Placebo

\begin{tabular}{|l|l|l|l|}
\hline Transition & $\begin{array}{l}\text { Placebo (No. per } \\
\text { Night) }\end{array}$ & $\begin{array}{l}\text { BJ (No. per } \\
\text { Night) }\end{array}$ & Wilcoxon \\
\hline W-NI & $14.1 \pm 2.0$ & $11.3 \pm 1.7$ & NS \\
W-N2 & $1.4 \pm 0.3$ & $0.6 \pm 0.2$ & 0.034 \\
W-N3 & 0.0 & 0.0 & NS \\
W-R & $0.3 \pm 0.1$ & 0.0 & 0.021 \\
N2/N3 to REM & $2.5 \pm 0.3$ & $3.7 \pm 0.6$ & 0.023 \\
All NREM to REM & $5.0 \pm 0.8$ & $6.4 \pm 1.1$ & 0.033 \\
\hline
\end{tabular}

elevated by $\mathrm{BJ}\left(43176 \pm 8660 \mu \mathrm{V}^{2}\right.$ ominutes) relative to placebo (31335 $\pm 5807 \mu \mathrm{V}^{2}$ ominutes) in the C4:M1 derivation $\left(\mathrm{T}_{8}=\right.$ 2.31, $P=0.049$ ). A similar nonsignificant trend toward elevation by $\mathrm{BJ}$ ( $37310 \pm 8457 \mu \mathrm{V}^{2}$ ominutes) relative to placebo (28564 $\pm 5834 \mu \mathrm{V}^{2}$ ominutes) occurred in the $\mathrm{F} 4: \mathrm{M} 1$ derivation ( $\left.\mathrm{T}_{8}=2.11, P=0.067\right)$.

\section{Discussion}

Beetroot juice normalized objective measures of sleep quality in a cohort of COPD patients. Although BJ was without effects on total time spent in each of the polysomnographically defined sleep states, atypical sequences of events seen in the placebo condition (direct wake-toREMS transitions, transitions from wake to intermediate depth N2 sleep) were reduced in frequency by BJ. Sleep state sequences common to healthy sleep (NREMS-toREMS) were increased in frequency by BJ. Changes in sleep that unfold across the night in normal sleep (the decline in N3 slow wave activity, the increase in REMS as a percentage of time), were facilitated by BJ consumption. Collectively, the reduced frequency of wake-toREMS transitions and the increased frequency of NREMS-to-REMS transitions after BJ are indicative of normalization of the sequencing of events within the sleep cycle.

In addition, $\mathrm{O}_{2}$ saturation was elevated by $\mathrm{BJ}$, albeit only in periods of wakefulness after sleep onset. Because hypoxia is reported to elevate EEG power in the delta range during wakefulness, ${ }^{25-28}$ we hypothesized that effect of nitrate on $\mathrm{SpO}_{2}$ during wake would result in changes in the waking EEG spectra. The suppression of delta power during wakefulness by BJ administration is supportive of the concept that BJ promotes cerebral oxygenation, at least during intervals of wakefulness. These data indicate a potential therapeutic effect for bedtime $\mathrm{BJ}$ in the treatment of disordered sleep in COPD.
The principal challenge to intervention in COPD is to expel carbon dioxide and to deliver sufficient $\mathrm{O}_{2}$ into the circulation. Increasing accessory muscle tone to increase airflow, a mechanism used by healthy individuals in the face of exertion-induced increases in carbon dioxide production and $\mathrm{O}_{2}$ demand, is a compensatory strategy that COPD patients employ constitutively during sleep. ${ }^{2}$ Yet the respiratory challenges associated with recumbency and the skeletal muscle relaxation that occurs during sleep compromise accessory muscle function. ${ }^{1,29}$ The trade-off between the need to sleep and the need to employ accessory muscles is likely to negatively impact sleep quality in COPD: across polysomnographically monitored COPD subjects, the extent to which accessory muscles are activated during sleep is proportional to the severity of disruption of sleep. ${ }^{2}$ Adjunct therapeutic measures that improve the delivery of circulating oxygen or the efficiency of oxygen utilization may be beneficial to sleep in COPD.

Orally administered nitrate in BJ undergoes conversion to nitrite in the digestive tract, which then undergoes conversion to nitric oxide systemically, resulting in increased efficiency of oxygen delivery and aerobic respiration. ${ }^{6} \mathrm{BJ}$ elevates plasma nitrate acutely in COPD patients when administered during the day. ${ }^{8,10,11}$ As shown here, when $\mathrm{BJ}$ is administered at bedtime, the elevation of plasma nitrate is of sufficient duration to be detected in plasma samples taken the next morning. However, nitrite levels were not significantly higher compared to controls. Since plasma half-life of nitrate is approximately five to six hours but only 20-30 min for nitrite and the last dose of BJ was administered the night before and samples taken the morning after, this may explain the lack of difference in plasma nitrite between the groups. Moreover, we cannot fully exclude some oxidation of nitrite to nitrate during sample handling, which is a known methodological concern when handling these anions.

Previous observations of sleep fragmentation in $\mathrm{COPD}^{3,4}$ provide a target for therapeutic intervention. Although we did not compare the COPD population to a healthy age-matched control group, salient features of sleep pathology were present in the subjects (transitions from wake to $\mathrm{N} 2$ and vice versa and direct transitions from wake to REMS). These pathological events were reduced in frequency by consumption of BJ with nitrate as an active component. Wake-to-REMS transitions in untreated COPD may reflect a high REM propensity, as the 

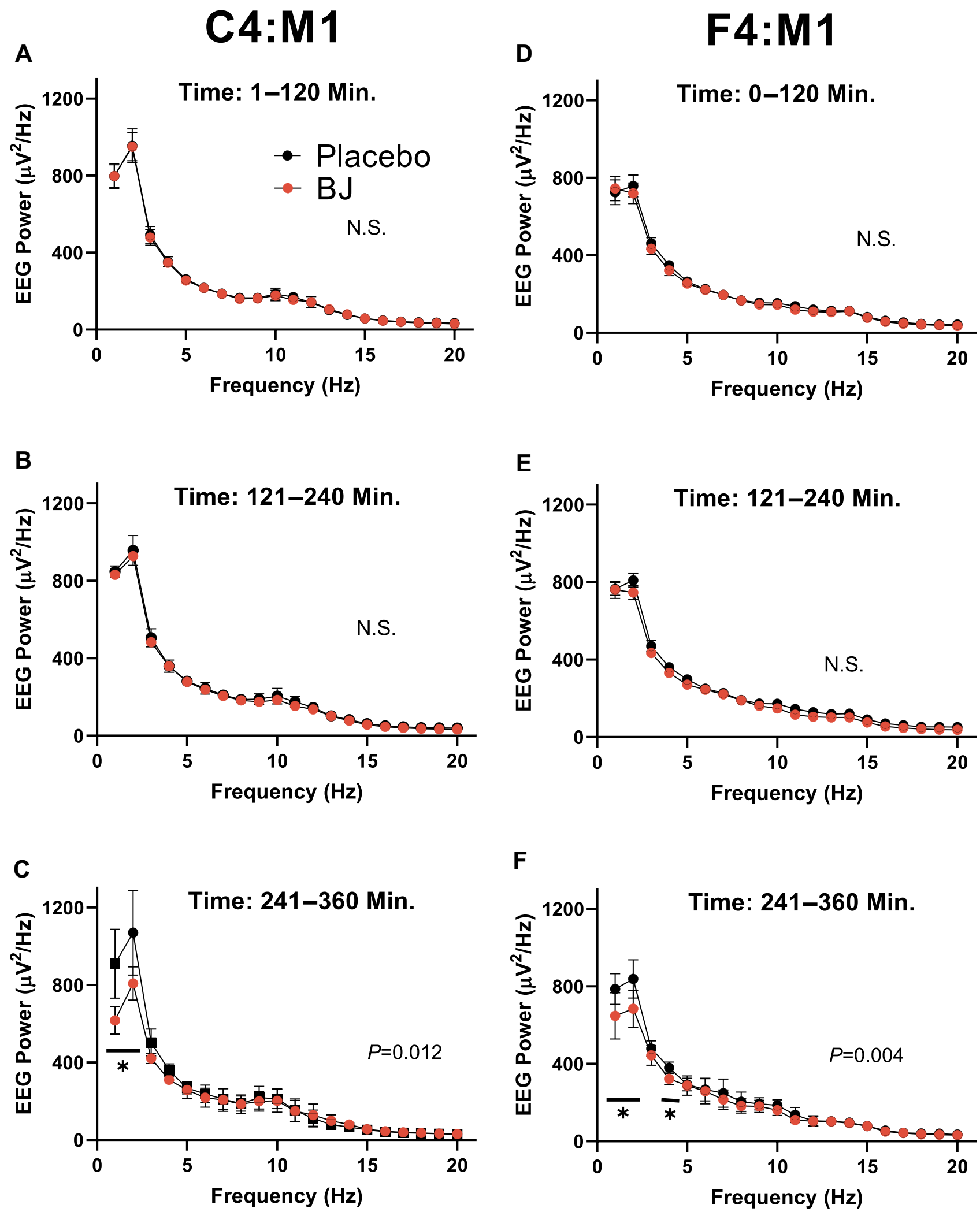

Figure 6 EEG spectral power during N3. (A-C) Data from the C4:MI derivation in the first, second, and third two-hour intervals (D-F) Data from the F4:MI derivation in the first, second, and third two-hour intervals. $P$-values refer to treatment $\dot{x}$ frequency interaction in repeated measured $A N O V A$. $* P<0.05$ beetroot juice (BJ) vs placebo value at same frequency, Fisher's LSD. Abbreviation: N.S., not significant. 
propensity for REMS accumulates during NREMS.$^{30}$ By analogy, sleep onset REM-sleep periods (SOREMPs) ${ }^{31}$ and NREMS fragmentation ${ }^{32}$ are seen in obstructive sleep apnea (OSA). In OSA, insufficient $\mathrm{O}_{2}$ delivery during sleep prevents the normal (typically hour-long) sequence of states from wake to light non-REMS (N1) to deeper non-REMS (N2/N3) to REMS. SOREMPs in OSA are a manifestation of elevated REMS propensity due to the inability to maintain normal state sequencing. The wake-to-REMS transitions in untreated COPD are likely to be analogous in their pathophysiological basis. Protracted NREMS cannot occur due to insufficient $\mathrm{O}_{2}$ delivery during sleep (possibly exacerbated by increased carbon dioxide); REMS pressure builds during repeatedly aborted wake/deep sleep transitions and is then manifested as wake-to-REMS transitions.

The dynamics of the NREMS EEG across the night, particularly the decline in EEG delta power in N3 sleep, reflect a discharge of homeostatic sleep need. ${ }^{33}$ Since per-epoch EEG power was equivalent in the first two-hour intervals of the $\mathrm{BJ}$ and placebo nights, but lower in the third two-hour interval of $\mathrm{BJ}$ relative to placebo, there may have been a greater discharge of sleep need under the influence of nitrate. Increased time spent in deep sleep, or increased state stability, might explain accelerated discharge. In the sleep of healthy young adults, it is typical for the NREMS states to occur sequentially in proportion to their depth $(\mathrm{N} 1 / \mathrm{N} 2 / \mathrm{N} 3)$ at sleep onset; transitions from wake to $\mathrm{N} 2$ are less frequent than wake to $\mathrm{N} 1 .^{33}$ Here, waketo-N2 transitions were more frequent after placebo (a total of 21 observed) than BJ (nine observed).

Collectively, suppression of slow wave activity in the wake state, and reduced numbers of both aborted wake-todeep sleep transitions and wake-to-REMS transitions in the $\mathrm{BJ}$ condition relative to the placebo led us to hypothesize that $\mathrm{O}_{2}$ delivery might be improved by BJ, allowing for more stable maintenance of sleep states. The statespecific dynamics of $\mathrm{SpO}_{2}$ values support this hypothesis only for episodes of WASO. During wake, accessory muscles can most effectively be put to use for $\mathrm{O}_{2}$ delivery; the loss of this mechanism due to sleep-related atonia may have nullified the effect of BJ.

\section{Limitations}

The study did not include data from non-COPD control subjects, but the counterbalanced, repeated measures design incorporated a control (placebo) condition to which the therapeutic intervention (BJ) could be compared. While it is clear from previous studies that COPD disrupts sleep, ${ }^{3,4}$ the absence of data from an age-matched healthy control population in the current study prevents us from quantifying the extent to which $\mathrm{BJ}$ ameliorates sleep deficits in COPD relative to the general population. No measures of daytime sleepiness or daytime performance on the morning after polysomnography were recorded, so it cannot be ascertained whether daytime functioning was positively impacted by this intervention. Furthermore, given that the $\mathrm{O}_{2}$ saturation during sleep was not altered by the intervention, it is uncertain whether any health outcomes might benefit in the longer term from this intervention. However, patients underwent a single exposure to $\mathrm{BJ}$ at the start of the night. Peak exposure (as measured by plasma nitrite) is within two to three hours of administration in awake, healthy subjects. ${ }^{34,35}$ Repeated exposures within the night or across nights might have more robust effects, as the effects of nitrate supplementation do not attenuate over at least two weeks of daily administration. ${ }^{34}$

Given the lack of change in oxygen saturation during sleep in the BJ condition relative to placebo, it is possible that the polysomnographic effect of $\mathrm{BJ}$ is due not to a clinically significant change in oxygen utilization or availability, but rather to a decrease in the awakening response to sleep-dependent hypoventilation after BJ; subjects may have continued to undergo NREMS even in the face of hypoxic conditions after BJ. As a significant percentage of COPD patients show signs of sleep hypoventilation, ${ }^{5,36}$ simultaneous recording of transcutaneous carbon dioxide and/or carboxyhemoglobin should be considered in future studies to address this possibility.

\section{Conclusion}

Dietary nitrate supplementation induced polysomnographic changes in patients suffering from COPD: (1) It abolished wake-to-REMS transitions and increased the likelihood of deep NREMS-to-REMS transitions. (2) It increased the amount of time spent in REMS in the latter portion of the night. (3) It precipitated an accelerated discharge of slow wave activity across the night. Collectively, these changes in objective sleep parameters are indicative of improved sleep quality and suggest that BJ can benefit sleep quality, if not tissue oxygenation, in COPD.

\section{Abbreviations}

AW, active wake; AASM, American Academy of Sleep Medicine; BJ, beetroot juice containing nitrate; IW, 
intermediate wake; OSA, obstructive sleep apnea; $\mathrm{SpO}_{2}$, oxygen saturation; QW, quiet wake; REMS, rapid eye movement sleep; SE, sleep efficiency; SOREMPs, sleep onset REM-sleep periods; N1, N2, N3, stages 1, 2, 3 nonrapid eye movement sleep; TST, total sleep time; WASO, wake after sleep onset.

\section{Acknowledgments}

This research was supported by Strategic Program for International Research Cooperation (SPIRE, project number 710022) at the University of Bergen. The authors wish to thank the nurses Jorun Haugen and Inger Åshild Krogh at the LHL Hospital Gardermoen, for their extra effort. Special thanks to Dr. Erlend Strand Gardsjord and Dr. Marit Rein Solhaug for their hospitality and generosity during data collection. This study was funded by the National Association for Heart and Lung Disease, Jessheim, Norway and by grants from the Norwegian Centre of Excellence in Home Mechanical Ventilation (grant number 4644398) and the National Competence Centre for Sleep Disorders, both at Haukeland University Hospital, Bergen, Norway. The abstract of this paper was presented at the Virtual SLEEP 2020 meeting of the Sleep Research Society/American Academy of Sleep Medicine as a poster presentation with interim findings. The poster's abstract was published as a supplement in the journal SLEEP: https://academic.oup.com/sleep/article-abstract/ 43/Supplement_1/A388/5846789?redirectedFrom=fulltext.

\section{Disclosure}

Nils Henrik Holmedahl reports grants from SovNo, Haukeland University Hospital, during the conduct of the study; and has received a research grant from Novartis, Norway, outside the submitted work. Eddie Weitzberg holds patents related to the therapeutic use of nitrate and nitrite and is a shareholder in Heartbeet Ltd. The authors report no other conflicts of interest in this work.

\section{References}

1. Johnson MW, Remmers JE. Accessory muscle activity during sleep in chronic obstructive pulmonary disease.. J Appl Physiol Respir Environ Exerc Physiol. 1984;57(4):1011-1017. doi:10.1152/jappl.1984.57.4.1011

2. Redolfi S, Grassion L, Rivals I, et al. Abnormal Activity of Neck Inspiratory Muscles during Sleep as a Prognostic Indicator in Chronic Obstructive Pulmonary Disease. Am J Respir Crit Care Med. 2020;201 (4):414-422. doi:10.1164/rccm.201907-1312OC

3. Valipour A, Lavie P, Lothaller H, Mikulic I, Burghuber OC. Sleep profile and symptoms of sleep disorders in patients with stable mild to moderate chronic obstructive pulmonary disease. Sleep Med. 2011;12 (4):367-372. doi:10.1016/j.sleep.2010.08.017
4. Fleetham J, West P, Mezon B, Conway W, Roth T, Kryger M. Sleep, arousals, and oxygen desaturation in chronic obstructive pulmonary disease. The effect of oxygen therapy. Am Rev Respir Dis. 1982;126 (3):429-433. doi:10.1164/arrd.1982.126.3.429

5. Holmedahl NH, Overland B, Fondenes O, Ellingsen I, Hardie JA. Sleep hypoventilation and daytime hypercapnia in stable chronic obstructive pulmonary disease. Int J Chron Obstruct Pulmon Dis. 2014;9:265-275. doi:10.2147/COPD.S57576

6. Lundberg JO, Weitzberg E, Gladwin MT. The nitrate-nitrite-nitric oxide pathway in physiology and therapeutics. Nat Rev Drug Discov. 2008;7(2):156-167. doi:10.1038/nrd2466

7. Larsen FJ, Weitzberg E, Lundberg JO, Ekblom B. Dietary nitrate reduces maximal oxygen consumption while maintaining work performance in maximal exercise. Free Radic Biol Med. 2010;48 (2):342-347. doi:10.1016/j.freeradbiomed.2009.11.006

8. Shepherd AI, Wilkerson DP, Dobson L, et al. The effect of dietary nitrate supplementation on the oxygen cost of cycling, walking performance and resting blood pressure in individuals with chronic obstructive pulmonary disease: A double blind placebo controlled, randomised control trial. Nitric Oxide. 2015;48:31-37. doi:10.1016/j. niox.2015.01.002

9. Leong $\mathrm{P}$, Basham JE, Yong $\mathrm{T}$, et al. A double blind randomized placebo control crossover trial on the effect of dietary nitrate supplementation on exercise tolerance in stable moderate chronic obstructive pulmonary disease. BMC Pulm Med. 2015;15:52. doi:10.1186/ s12890-015-0057-4

10. Kerley CP, James PE, McGowan A, Faul J, Cormican L. Dietary nitrate improved exercise capacity in COPD but not blood pressure or pulmonary function: a 2 week, double-blind randomised, placebocontrolled crossover trial. Int J Food Sci Nutr. 2019;70(2):222-231. doi:10.1080/09637486.2018.1492521

11. Berry MJ, Justus NW, Hauser JI, et al. Dietary nitrate supplementation improves exercise performance and decreases blood pressure in COPD patients. Nitric Oxide. 2015;48:22-30. doi:10.1016/j.niox.2014.10.007

12. Susswein AJ, Katzoff A, Miller N, Hurwitz I. Nitric oxide and memory. Neuroscientist. 2004;10(2):153-162. doi:10.1177/ 1073858403261226

13. Toda N, Ayajiki K, Okamura T. Cerebral blood flow regulation by nitric oxide: recent advances. Pharmacol Rev. 2009;61(1):62-97. doi:10.1124/pr.108.000547

14. Hoiland RL, Caldwell HG, Howe CA, et al. Nitric oxide is fundamental to neurovascular coupling in humans. J Physiol. 2020;598 (21):4927-4939. doi:10.1113/JP280162

15. Gautier-Sauvigne S, Colas D, Parmantier P, et al. Nitric oxide and sleep. Sleep Med Rev. 2005;9(2):101-113. doi:10.1016/j. smrv.2004.07.004

16. Chen Z-Q, Mou R-T, Feng D-X, Wang Z, Chen G. The role of nitric oxide in stroke. Med Gas Res. 2017;7(3):194-203. doi:10.4103/20459912.215750

17. Wightman EL, Haskell-Ramsay CF, Thompson KG, et al. Dietary nitrate modulates cerebral blood flow parameters and cognitive performance in humans: A double-blind, placebo-controlled, crossover investigation. Physiol Behav. 2015;149:149-158. doi:10.1016/j. physbeh.2015.05.035

18. Presley TD, Morgan AR, Bechtold E, et al. Acute effect of a high nitrate diet on brain perfusion in older adults. Nitric Oxide. 2011;24 (1):34-42. doi:10.1016/j.niox.2010.10.002

19. Gilchrist M, Winyard PG, Fulford J, Anning C, Shore AC, Benjamin N. Dietary nitrate supplementation improves reaction time in type 2 diabetes: development and application of a novel nitrate-depleted beetroot juice placebo. Nitric Oxide. 2014;40:67-74. doi:10.1016/j. niox.2014.05.003

20. Holmedahl NH, Fjeldstad O-M, Engan H, Saxvig IW, Gronli J. Validation of peripheral arterial tonometry as tool for sleep assessment in chronic obstructive pulmonary disease. Sci Rep. 2019;9 (1):19392. doi:10.1038/s41598-019-55958-2 
21. Ormesher L, Myers JE, Chmiel C, et al. Effects of dietary nitrate supplementation, from beetroot juice, on blood pressure in hypertensive pregnant women: A randomised, double-blind, placebo-controlled feasibility trial. Nitric Oxide. 2018;80:37-44. doi:10.1016/j. niox.2018.08.004

22. Iber C, Ancoli-Israel S, Chesson AL, Quan SF. The AASM Manual for the Scoring of Sleep and Associated Events. Rules, Terminology and Technical Specifications. American academy of sleep medicine; 2007.

23. Berry RB, Budhiraja R, Gottlieb DJ, et al. Rules for scoring respiratory events in sleep: update of the 2007 AASM Manual for the Scoring of Sleep and Associated Events. Deliberations of the Sleep Apnea Definitions Task Force of the American Academy of Sleep Medicine.. J Clin Sleep Med. 2012;8(5):597-619. doi:10.5664/jcsm.2172

24. Jansson EÅ, Huang L, Malkey R, et al. A mammalian functional nitrate reductase that regulates nitrite and nitric oxide homeostasis. Nat Chem Biol. 2008;4(7):411-417. doi:10.1038/nchembio.92

25. Saletu B, Hitzenberger G, Grunberger J, et al. Double-blind, placebocontrolled, pharmacokinetic and -dynamic studies with 2 new formulations of piracetam (infusion and sirup) under hypoxia in man.. Int J Clin Pharmacol Ther. 1995;33(5):249-262.

26. Saletu B, Grunberger J, Anderer P, Linzmayer L. Effects of the novel neuroprotective agent, riluzole, on human brain function and behavior: II. Double-blind, placebo-controlled EEG mapping and psychometric studies under hypoxia.. Methods Find Exp Clin Pharmacol. 1996;18(1):67-81.

27. Guthrie RD, Knauss TA, Haberkern CM, Sumi SM, Woodrum DE. Power spectral analysis of the neonatal primate electroencephalogram during acute hypoxemia. Pediatr Res. 1982;16(Supplement):30-34. doi:10.1203/00006450-198201001-00006

28. Burykh ÉA. Interaction between changes in local and temporospatial spectral EEG characteristics during exposure of humans to hypoxia. Neurosci Behav Physiol. 2007;37(2):133-146. doi:10.1007/s11055007-0161-x
29. McNicholas WT. Impact of sleep on respiratory muscle function. Monaldi arch Chest dis. 2002;57(5-6):277-280.

30. Benington JH, Heller HC. REM-sleep timing is controlled homeostatically by accumulation of REM-sleep propensity in non-REM sleep. Am J Physiol. 1994;266(6 Pt 2):R1992. doi:10.1152/ ajpregu.1994.266.6.R1992

31. Seneviratne U, Puvanendran K. Excessive daytime sleepiness in obstructive sleep apnea: prevalence, severity, and predictors. Sleep Med. 2004;5(4):339-343. doi:10.1016/j.sleep.2004.01.021

32. Greenberg H, Lakticova V, Scharf SM. Obstructive Sleep Apnea: clinical Features, Evaluation, and Principles of Management. In: Kryger MH, Roth T, Dement WC, editors. Principles and Practice of Sleep Medicine. 6th ed. W.B. Saunders; 2017:1110-1124.

33. Borbely AA, Achermann P. Sleep homeostasis and models of sleep regulation. In: Kryger MH, Roth T, Dement WC, editors. Principles and Practice of Sleep Medicine. 3rd ed. W.B. Saunders; 2004:377390.

34. Jones AM, Bailey SJ, Vanhatalo A. Dietary nitrate and O(2) consumption during exercise. Med Sport Sci. 2012;59:29-35. doi:10.1159/000342062

35. Wylie LJ, Kelly J, Bailey SJ, et al. Beetroot juice and exercise: pharmacodynamic and dose-response relationships. J Appl Physiol. 2013;115(3):325-336. doi:10.1152/japplphysiol.00372.2013

36. Ellingsen I, Fondenes O, Overland B, Holmedahl NH. The severity of sleep hypoventilation in stable chronic obstructive pulmonary disease. Sleep Breath. 2020. doi:10.1007/s11325-020-02097-y
Nature and Science of Sleep

\section{Publish your work in this journal}

Nature and Science of Sleep is an international, peer-reviewed, open access journal covering all aspects of sleep science and sleep medicine, including the neurophysiology and functions of sleep, the genetics of sleep, sleep and society, biological rhythms, dreaming, sleep disorders and therapy, and strategies to optimize healthy sleep.

\section{Dovepress}

The manuscript management system is completely online and includes a very quick and fair peer-review system, which is all easy to use. Visit http://www.dovepress.com/testimonials.php to read real quotes from published authors. 